\title{
Cosmology of a brane radiating gravitons into the extra dimension
}

\author{
David Langlois, Lorenzo Sorbo, María Rodríguez-Martínez \\ GReCO, Institut d'Astrophysique de Paris, \\ Centre National de la Recherche Scientifique, \\ 98bis Boulevard Arago, 75014 Paris, France
}

(October 25, 2018)

\begin{abstract}
We study in a self-consistent way the impact of the emission of bulk gravitons on the (homogeneous) cosmology of a three-brane embedded in a fivedimensional spacetime. In the low energy regime, we recover the well known result that the bulk affects the Friedmann equation only via a radiation-like term $\mathcal{C} / a^{4}$, called dark or Weyl radiation. By contrast, in the high energy regime, we find that the Weyl parameter $\mathcal{C}$ is no longer constant but instead grows very rapidly as $\mathcal{C} \propto a^{4}$. As a consequence, the value of $\mathcal{C}$ today is not a free parameter as usually considered but is a fixed number, which, generically, depends only on the number of relativistic degrees of freedom at the high/low energy transition. Our estimated amount of Weyl radiation satisfies the present nucleosynthesis bounds.
\end{abstract}

\section{INTRODUCTION}

In the last couple of years, considerable attention has been paid to a new type of models with extra dimensions, for which ordinary matter is confined on a three-dimensional space called brane. Although a lot of effort has been devoted to investigate the signatures of extra-dimensions in gravity tests and collider experiments, these potentially new effects will 
be inaccessible if the higher-dimensional fundamental mass is too high. Cosmology would then be the last hope to detect an extra-dimensional signature, via for instance the Cosmic Microwave Background Radiation (CMBR) anisotropies [1], because of the high energies reached in the very early universe.

The simplest viable model of brane cosmology that takes into account the self-gravity of the brane is the cosmological extension [2] of the Randall-Sundrum (RS) setup [3] and consists of a $Z_{2}$-symmetric (i.e. mirror symmetric) three-brane, with a tension $\lambda$ and containing ordinary cosmological matter with energy density $\rho$, embedded in an empty five-dimensional spacetime endowed with a (negative) cosmological constant $\Lambda \equiv-6 \mu^{2}$. In this context, one can solve explicitly [4], for any equation of state of brane matter, the five-dimensional Einstein's equations

$$
G_{A B}+\Lambda g_{A B}=\kappa^{2} T_{A B}
$$

where $\kappa^{2} \equiv M^{-3}$ is the five-dimensional gravitational coupling and where the energymomentum tensor here vanishes everywhere except on the brane. Imposing the RS condition [3] $\kappa^{2} \lambda=6 \mu$, this leads to the generalized Friedmann law [4 6]

$$
H^{2}=\frac{\kappa^{4}}{18} \lambda \rho+\frac{\kappa^{4}}{36} \rho^{2}+\frac{\mathcal{C}}{a^{4}}
$$

where $H \equiv \dot{a} / a$ is the expansion rate and $\mathcal{C}$ is an arbitrary constant. Although the first term on the right-hand side, with the identification $\kappa^{4} \lambda / 6=\kappa^{2} \mu \equiv 8 \pi G=\bar{M}_{p}^{-2}$, agrees with the familiar Friedmann equation, the two additional terms induce a deviation from the usual law. First, there is a term quadratic in $\rho$ [7], which dominates the usual linear term in the high energy regime, when $\rho \gg \lambda$. Second, one finds a radiation-like term proportional to the integration constant $\mathcal{C}$, which we will call here the Weyl parameter since it is related to the bulk Weyl tensor [6] and which is formally analogous to the Schwarzschild mass which arises as an integration constant when solving the vacuum Einstein equations with spherical symmetry. Indeed, the five-dimensional spacetime metric is Schwarzschild-Anti de Sitter (Sch-AdS) which can be written in the manifestly static form [5] 


$$
d s^{2}=-f(r) d T^{2}+\frac{d r^{2}}{f(r)}+r^{2} d \mathbf{x}^{2}, \quad f(r)=\mu^{2} r^{2}-\frac{\mathcal{C}}{r^{2}}
$$

and the 'radial' coordinate $r$ of the brane can be identified with its scale factor $a$. When the bulk spacetime is empty, $\mathcal{C}$ is necessarily constant in time. This can be seen as a generalization to five dimensions of Birkoff's theorem [8].

In this setup, one recovers the usual conservation law $\dot{\rho}+3 H(\rho+p)=0$. This picture is however oversimplified in the sense that, if the assumption of homogeneity is justified on average, there exist fluctuations on small scales, which create gravitational waves that can escape into the bulk. In the language of particle physics, one would say that interactions of brane particles will generate bulk gravitons. If this question has been analysed in detail in the context of flat compact extra-dimensions (see e.g. [9]), it has received so far much less attention in the RS context [10], the main difficulty being to take into account the back reaction of the graviton flow on the bulk metric. The purpose of the present work is precisely to present a treatment that combines self-consistently the emission of gravitons with the surrounding spacetime in which our brane-universe is moving.

\section{BRANE IN A RADIATIVE SPACETIME}

In order to model the bulk spacetime metric, we generalize to five dimensions the fourdimensional Vaidya's metric [11], which is used in general relativity to describe the spacetime surrounding a radiating star. Our metric ansatz is given by

$$
d s^{2}=-f(r, v) d v^{2}+2 d r d v+r^{2} d \mathbf{x}^{2}, \quad f(r, v)=\mu^{2} r^{2}-\frac{\mathcal{C}(v)}{r^{2}} .
$$

If $\mathcal{C}$ does not depend on $v$, the above metric is simply a rewriting of the Sch-AdS metric (3) with the change of coordinate $v=T+\int d r / f(r)$. In all cases, $v$ is a null coordinate since the spacetime trajectory defined by $v=$ const (and $x^{i}=$ const) is light-like. In Schwarzschild spacetime, it would correspond to ingoing radial light rays. Note that, strictly speaking, the Vaidya spacetime corresponds to an outgoing radiation flow, whereas in our case, we are interested in an ingoing radial flow because the brane, which emits the radiation, is in 
some sense located at the largest radius of spacetime (when getting away from the brane, one moves radially 'inwards', as can be deduced from the junction conditions (8)).

In the above spacetime $(\mathbb{\Psi})$, the trajectory of the brane can be expressed in terms of its coordinates $v(t)$ and $r(t)$ as functions of the proper time $t$ (which is also the cosmic time). The normalization of the velocity vector $\left(u^{A}=\{\dot{v}, \dot{r}, \mathbf{0}\}\right.$, where a dot stands for a derivation with respect to $t$ ) implies

$$
\dot{v}=\frac{\dot{r}+\sqrt{f+\dot{r}^{2}}}{f},
$$

assuming that the brane is always in the region of spacetime where $f>0$ (i.e. outside the horizon in the Schwarzschild case).

Similarly to its four-dimensional counterpart, it is easy to verify that the metric (舟) is a solution of Einstein's equations (1) with a bulk energy-momentum tensor of the form

$$
T_{A B}=\sigma k_{A} k_{B}
$$

where $k^{A}$ is a (here ingoing) null vector. Our model thus corresponds to a simplified picture, where the bulk gravitons are supposed to escape 'radially' from the brane. Choosing to normalize it so that $k_{A} u^{A}=1$, the only non-vanishing component is $k^{r}\left(=k_{v}\right)=1 / \dot{v}$. The quantity $\sigma$ represents, for a brane observer, the energy flux of the bulk gravitons and is related, via Einstein's equations (11), to the variation of the Weyl parameter, according to the expression

$$
\frac{d \mathcal{C}}{d v}=\frac{2 \kappa^{2} \sigma}{3} r^{3}\left(\dot{r}-\sqrt{f+\dot{r}^{2}}\right)^{2}
$$

The Einstein equations being solved in the bulk, it remains to impose at the brane location the Israel junction conditions, which relate the jump of the extrinsic curvature tensor for the brane $K_{A B}=h_{A}^{C} \nabla_{C} n_{B}$ ( $n^{A}$ is the unit vector normal to the brane and $h_{A B}=g_{A B}-n_{A} n_{B}$ the induced metric) to the brane energy-momentum tensor $\tau_{A B}$, namely 团

$$
\left[K_{A B}\right]=\kappa^{2}\left(\tau_{A B}-\frac{1}{3} \tau h_{A B}\right)
$$


Here, $\tau_{A B}=\left(\rho_{b}+p_{b}\right) u_{A} u_{B}+p_{b} h_{A B}$, where $\rho_{b}=\lambda+\rho$ and $p_{b}=-\lambda+p$ are respectively the total energy density and pressure in the brane. The ordinary spatial components of the junction conditions yields (using $n_{A}=\{\dot{r},-\dot{v}, \mathbf{0}\}$ ) the expression

$$
\frac{\kappa^{2}}{6} \rho_{b}=\frac{\sqrt{f+\dot{r}^{2}}}{r},
$$

which implies the same unconventional Friedmann equation as in (2), with the only difference that $\mathcal{C}$ is a function of $v$ instead of a constant. The other components (along the time and the fifth dimension) yield another relation, which upon using (2) and its time derivative as well as (7), can be rewritten as

$$
\dot{\rho}_{b}+3 \frac{\dot{r}}{r}\left(\rho_{b}+p_{b}\right)=-2 \sigma .
$$

This equation simply expresses the fact that, from the point of view of the brane, the energy loss suffered by the brane exactly corresponds to the bulk radiation flow embodied in (6) (note that the right-hand side is very simple here because we have chosen to normalize $k^{A}$, and thus $\sigma$, with respect to the brane observers). There is a factor 2 because the brane is radiating on both sides, into two copies of the same bulk spacetime.

\section{PRODUCTION RATE OF BULK GRAVITONS}

In order to determine quantitatively the energy loss $\sigma$ we now need to evaluate the cross-section of the process $\psi+\bar{\psi} \rightarrow G$, where $\psi$ is a particle confined on the brane $(\bar{\psi}$ its antiparticle) and $G$ a bulk graviton, process which appears to be the dominant effect in the very early universe. To compute this cross-section, one can ignore the cosmological influence since the temperature $T$ of the brane particles is always much bigger than $H$ (indeed $H \sim T^{4} / M^{3}$ in the high energy regime and $H \sim T^{2} / \bar{M}_{p}$ in the low energy regime, and we always assume $T \ll M)$. A Minkowski background in the brane corresponds to the five-dimensional Randall-Sundrum solution [3]. Starting from the linear perturbations of the RS metric, 


$$
d s^{2}=\left(e^{-2 \mu|y|} \eta_{\mu \nu}+2 \kappa h_{\mu \nu}(x, y)\right) d x^{\mu} d x^{\nu}+d y^{2}
$$

in the RS gauge (i.e. $h_{\mu}^{\mu}=0$ and $\partial^{\mu} h_{\mu \nu}=0$ ), the gravitons correspond to the decomposition into (generalized) Kaluza-Klein (KK) modes,

$$
h_{\mu \nu}(x, y)=\int d m u_{m}(y) \phi_{\mu \nu}^{(m)}(x)
$$

where the modes $u_{m}(y)$ are given by [12]

$$
u_{m}(y)=\sqrt{\frac{m}{2 \mu}} \frac{J_{1}(m / \mu) N_{2}\left(m e^{\mu|y|} / \mu\right)-N_{1}(m / \mu) J_{2}\left(m e^{\mu|y|} / \mu\right)}{\sqrt{\left(J_{1}(m / \mu)\right)^{2}+\left(N_{1}(m / \mu)\right)^{2}}},
$$

and satisfy the normalization $\int d y e^{2 \mu|y|} u_{m}^{*}(y) u_{m^{\prime}}(y)=\delta\left(m-m^{\prime}\right)$. The $h_{\mu \nu}$ have been defined so that the kinetic part of the graviton action is of the canonical form $S_{k i n}=$ $-(1 / 2) \int d m \int d^{4} x\left(\partial \phi_{m}\right)^{2}$. The interaction between brane matter (living at $y=0$ ) and the bulk gravitons is described by the action

$$
\mathcal{S}_{i n t}=\kappa \int d m u_{m}(0) \int d^{4} x \tau^{\mu \nu} \phi_{\mu \nu}^{(m)}
$$

which means that the effective coupling constant for the 'canonical' $\mathrm{KK}$ modes $\phi_{m}$ is $\kappa\left|u_{m}(0)\right|$. For the modes $m \gg \mu,(13)$ gives us $\left|u_{m}(0)\right| \simeq 1 / \sqrt{\pi}$.

The interaction Lagrangian (14) gives us the explicit coupling between any bulk KK graviton and brane matter, from which one can derive the amplitude for the scattering of brane particles leading to a KK emission. At this stage, the calculation is quite analogous to the procedure already described in the context of flat extra dimensions [13, the only difference being the coupling constant in (14). Using those results, one finds that the spin and particle-antiparticle averaged squared amplitude (neglecting the mass of the incoming particles) is given here by

$$
\sum|\mathcal{M}|^{2}=\kappa^{2}\left|u_{m}(0)\right|^{2} A \frac{s^{2}}{8}
$$

with $s=\left(p_{1}+p_{2}\right)^{2}\left(p_{1}\right.$ and $p_{2}$ being the incoming 4-momenta of the scattering particles), and with $A_{s}=2 / 3$ for scalar-scalar scattering, $A_{f}=1$ for fermion-fermion scattering, and $A_{v}=4$ for photon-photon scattering. 
Going back to cosmology, the brane matter energy density deficit corresponds to the energy density created in the form of bulk gravitons, which can be expressed, after integrating the Boltzmann equation over momentum space, as

$$
\frac{d \rho}{d t}+3 H(\rho+p)=-\int d m \int \frac{d^{3} p_{m}}{(2 \pi)^{3}} \mathbf{C}_{m}
$$

where the collision term associated with the scattering process described above is given by

$$
\mathbf{C}[f]=\frac{1}{2} \int \frac{d^{3} p_{1}}{(2 \pi)^{3} 2 E_{1}} \frac{d^{3} p_{2}}{(2 \pi)^{3} 2 E_{2}} \sum|\mathcal{M}|^{2} f_{1} f_{2}(2 \pi)^{4} \delta^{(4)}\left(p_{1}+p_{2}-p_{m}\right)
$$

with the Fermi/Bose distribution functions $f_{i}=1 /\left(e^{E_{i} / T} \pm 1\right)$, while $p_{m}$ is the fourmomentum of the created bulk graviton. We will assume that the temperature satisfies $T \gg \mu$ (this is an excellent approximation in the early universe for a large range of values

for $\mu$ since the only constraint, from gravity experiments, is $\mu \gtrsim 10^{-3} \mathrm{eV}$ ). The typical energy of gravitons created by brane particles will be of the order of $T$, so that most of the contribution to the energy loss will come from 'heavy' gravitons satisfying $m \gg \mu$. Therefore, we simplify the calculation by directly replacing $u_{m}(0)$ by $1 / \sqrt{\pi}$ in the right-hand side of (16), thus introducing only a slight error due to the very light KK modes $(m \lesssim \mu)$. One can then perform explicitly the integration to find

$$
\dot{\rho}+4 H \rho=-\left[\frac{315 \zeta(9 / 2) \zeta(7 / 2)}{512 \pi^{3}}\right] \hat{g}(T) \kappa^{2} T^{8},
$$

where we have introduced an effective number of degrees of freedom (relevant for bulk graviton production) defined by

$$
\hat{g}(T)=\left((2 / 3) g_{s}+4 g_{v}+\left(1-2^{-7 / 2}\right)\left(1-2^{-5 / 2}\right) g_{f}\right)
$$

where $g_{s}, g_{f}$ and $g_{v}$ are the number of relativistic degrees of freedom, at temperature $T$, for particles of respectively spin $0,1 / 2$ and 1 .

\section{COUPLED COSMOLOGICAL EVOLUTION}

We now combine all the above results in order to get a dynamical system describing the coupled evolution of the brane energy density, of the brane scale factor and of the Weyl 
parameter.

In the radiation era, on which we focus, the energy density loss rate $\sigma$ can be rewritten as

$$
\sigma=\frac{\alpha}{12} \kappa^{2} \rho^{2}, \quad \alpha=\frac{212625}{64 \pi^{7}} \zeta(9 / 2) \zeta(7 / 2) \frac{\hat{g}}{g_{*}^{2}}
$$

after use of the relation $\rho=\left(\pi^{2} / 30\right) g_{*} T^{4}$, where $g_{*}=g_{s}+g_{v}+(7 / 8) g_{f}$ is the total effective number of relativistic degrees of freedom in the energy density. If all degrees of freedom of the standard model are relativistic, $g_{*}=106.75$ and $\hat{g} \simeq 166.21$ so that $\alpha \simeq 0.019$. Introducing the dimensionless quantities $\hat{\rho}=\rho / \lambda, \hat{H}=H / \mu, \hat{t}=\mu t$, the equation governing the evolution of the energy density now reads

$$
\frac{d \hat{\rho}}{d \hat{t}}+4 \hat{H} \hat{\rho}=-\alpha \hat{\rho}^{2}
$$

whereas the Friedmann equation takes the form

$$
\hat{H}^{2}=2 \hat{\rho}+\hat{\rho}^{2}+\frac{\hat{\mathcal{C}}}{a^{4}}
$$

with the dimensionless Weyl parameter $\hat{\mathcal{C}}=\mathcal{C} / \mu^{2}$. The final equation we need is that governing the evolution of the Weyl parameter, which is given by

$$
\frac{d \hat{\mathcal{C}}}{d \hat{t}}=2 \alpha a^{4} \hat{\rho}^{2}(1+\hat{\rho}-\hat{H})
$$

It is obtained by writing $\dot{\mathcal{C}}=\dot{v}(d \mathcal{C} / d v)$ and by combining ([7), (5) and the junction condition (2). Note that one must always have $\hat{H}<1+\hat{\rho}$ as a consequence of our previous assumption $f>0$.

The coupled system consisting of (21), (22) and (23) can be analysed both numerically and, in some specific regimes, analytically. In the high energy regime $\hat{\rho} \gg 1$, the system approaches an asymptotic behaviour characterized by a rapid growth of the Weyl parameter due to an abundant production of bulk gravitons:

$$
\hat{\mathcal{C}} \sim \frac{\alpha}{4+\alpha} a^{4}, \quad a \sim t^{1 /(4+\alpha)}
$$


One thus sees that the evolution of the scale factor is different from the situation where the graviton outflow is ignored, in which case one finds, in the high energy regime, $a \propto t^{1 / 4}$. However, since $\alpha$ is very small for realistic cases, the power law is essentially the same.

In the low energy $(\hat{\rho} \ll 1)$ radiation era, one recovers the conventional evolution $a \sim t^{1 / 2}$ and the Weyl parameter approaches a constant value. Not surprisingly, this means that, during the late conventional cosmological evolution, the production of bulk gravitons can be safely ignored.

However, what is much more remarkable is that the asymptotic value for $\hat{\mathcal{C}}$ is determined quantitatively from the evolution of the brane-bulk system in the radiation phase. This asymptotic value for $\hat{\mathcal{C}}$ can be estimated analytically. If the present description is valid deep enough in the high energy regime, one finds

$$
\epsilon_{W} \equiv \frac{\rho_{W e y l}}{\rho_{\text {rad }}}=\frac{\hat{\mathcal{C}}}{2 a^{4} \hat{\rho}} \rightarrow \frac{\alpha}{4},
$$

after the high/low energy transition. It must be stressed that $\epsilon_{W}$ depends only on the number of relativistic degrees of freedom at the high/low energy transition, and, in particular, is independent of the five-dimensional Planck mass.

The ratio $\epsilon_{W}$, extrapolated at later times (i.e. taking into account the variation of $g_{*}$ ), is constrained by the number of additional relativistic degrees of freedom allowed during nucleosynthesis [14], which is usually expressed as the number of additional light neutrino species $\Delta N_{\nu}$. A typical bound $\Delta N_{\nu} \lesssim 1$ implies $\epsilon_{W} \lesssim 8 \times 10^{-2}$ at nucleosynthesis, whereas our model yields the value

$$
\epsilon_{W} \simeq 2 \times 10^{-3}\left(\frac{\hat{g}\left(T_{\mathrm{t}}\right)}{166.21}\right)\left(\frac{g_{*}\left(T_{n u c l}\right)}{10.75}\right)^{1 / 3}\left(\frac{106.75}{g_{*}\left(T_{\mathrm{t}}\right)}\right)^{7 / 3}
$$

where $T_{\mathrm{t}}$ is the temperature at the transition between the high and low energy regimes.

It is rather striking that the Weyl contribution which we have estimated turns out to be just below the present observational bounds. Given that forthcoming observations like the precise measurement of CMBR anisotropies might improve significantly the above bound [15], this opens the fascinating perspective that the simplest category of brane cosmology 
models could be confronted soon to observational tests at the level of homogeneous cosmology, and not only from cosmological perturbations. 


\section{REFERENCES}

[1] D. Langlois, Phys. Rev. Lett. 86, 2212 (2001) arXiv:hep-th/0010063].

[2] C. Csaki, M. Graesser, C. Kolda and J. Terning, Phys. Lett. B 462, 34 (1999) arXiv:hepph/9906513]; J. M. Cline, C. Grojean and G. Servant, Phys. Rev. Lett. 83, 4245 (1999) arXiv:hep-ph/9906523.

[3] L. Randall and R. Sundrum, Phys. Rev. Lett. 83 (1999) 4690 arXiv:hep-th/9906064.

[4] P. Binétruy, C. Deffayet, U. Ellwanger and D. Langlois, Phys. Lett. B 477 (2000) 285 arXiv:hep-th/9910219].

[5] P. Kraus, JHEP 9912 (1999) 011 arXiv:hep-th/9910149.

[6] T. Shiromizu, K. Maeda and M. Sasaki, Phys. Rev. D 62, 024012 (2000) arXiv:grqc/9910076.

[7] P. Binétruy, C. Deffayet and D. Langlois, Nucl. Phys. B 565 (2000) 269 arXiv:hepth/9905012].

[8] P. Bowcock, C. Charmousis and R. Gregory, Class. Quant. Grav. 17, 4745 (2000) arXiv:hep-th/0007177.

[9] L. J. Hall and D. R. Smith, Phys. Rev. D 60, 085008 (1999) arXiv:hep-ph/9904267;

S. Hannestad, Phys. Rev. D 64, 023515 (2001) arXiv:hep-ph/0102290.

[10] A. Hebecker and J. March-Russell, Nucl. Phys. B 608, 375 (2001) arXiv:hepph/0103214.

[11] P.C. Vaidya, Nature 171, 260 (1953); R.W. Lindquist, R.A. Schwartz, C.W. Misner, Phys. Rev. 137, B1360 (1965).

[12] J. Garriga and T. Tanaka, Phys. Rev. Lett. 84 (2000) 2778 arXiv:hep-th/9911055.

[13] G. F. Giudice, R. Rattazzi and J. D. Wells, Nucl. Phys. B 544, 3 (1999) arXiv:hep- 
ph/9811291]; T. Han, J. D. Lykken and R. J. Zhang, Phys. Rev. D 59, 105006 (1999) arXiv:hep-ph/9811350.

[14] K. A. Olive, G. Steigman and T. P. Walker, Phys. Rept. 333 (2000) 389 arXiv:astro $\mathrm{ph} / 9905320$.

[15] R. E. Lopez, S. Dodelson, A. Heckler and M. S. Turner, Phys. Rev. Lett. 82, 3952 (1999) arXiv:astro-ph/9803095. 